\title{
Intelligent Traffic Management and Load Balance Based on Spike ISDN-IoT
}

\author{
Nadia Adnan Shiltagh Al-Jamali,Member, IEEE, and Hamed S. Al-Raweshidy,Senior, IEEE
}

\begin{abstract}
An Intelligent Software Defined Network (ISDN) based on an intelligent controller, can manage and control the network in a remarkable way. In this paper, a methodology is proposed to estimate the packet flow at the sensing plane in the Software Defined Network-Internet of Things (SDN-IoT) based on a Partial Recurrent Spike Neural Network (PRSNN) congestion controller, to predict the next step ahead of packet flow and thus, reduce the congestion that may occur. That is, the proposed model (Spike ISDN-IoT) is enhanced with a congestion controller. This controller works as a proactive controller in the proposed model. In addition, we propose another intelligent clustering controller based on an artificial neural network, which operates as a reactive controller, to manage the clustering in the sensing area of the Spike ISDN-IoT. Hence, an intelligent queuing model is introduced to manage the flow table buffer capacity in the data plane of the spike ISDN-IoT network, such that the Quality of Service (QoS) of the whole network is improved. A modified training algorithm is introduced to train the PRSNN to adjust its weight and threshold in the hidden and output layers in a parallel manner. The simulation results demonstrate that the QoS is improved by $(\mathbf{1 4 . 3 6 \%})$ when using PRSNN as a congestion controller, as compared with a convolutional neural network (CNN).
\end{abstract}

Index Terms-Partial Recurrent Spike NN, cluster head, SDNIoT, traffic load prediction, Quality of Service.

\section{$\mathbf{T}$} HE concept of the Internet of Things (IoT) has been made a reality by the creation of Wireless Sensor Networks (WSNs), which have the capability of monitoring or controlling different applications across the connectivity of the Internet. The basic idea of IoT is to enable real objects that are inserted with sensors, actuators, and network connectivity to accumulate and shuffle data among themselves in a cooperative way [1]. In other words, the IoT can be described by this formula Things $+($ Intelligence + Network $=$ IoT) [2]. Many applications in the field of networks and the Internet require high speed, accuracy, security, and a high quality of services in the transfer of data. Accordingly, many solutions to enhance the Internet and computer networks with a high quality of services have been proposed, one of which is SDN-IoT. In an SDN, the data plane basically consists of a number of switches, routers, and gateways, while the control plane is

Manuscript received November 20,2019.(Corresponding author: Nadia A. Al-Jamali)

Nadia Adnan Shiltagh Al-Jamli with the department of Computer Engineering, University of Baghdad, Baghdad, Iraq. and with the department of Electronic and Computer Engineering, Brunel University London, London UB8 3PH, U.K.(e-mail: nadiaadnanshiltagh.aljamli@brunel.ac.uk).

Hamed S. Al-Raweshidy is with the department of Electronic and Computer Engineering, Brunel University London, London UB8 3PH, U.K. (e-mail: hamed.al-raweshidy@brunel.ac.uk). responsible for taking the decisions for each node in the data plane using a southbound interface. [3]. The SDN controller has two interfaces: southbound and northbound. The role of the southbound interface has been described above, while the northbound one is tasked with providing services in the form of applications on the top of the SDN controller [4]. Proficient protocol that enables the controller in SDN network reaching the switches and routers in the data plane is referred to as OpenFlow protocol [5]. OpenFlow has been adopted in a wide range of SDN applications such as Wide Area Networks (WAN), Internet exchange point, data center networks and cellular networks [6].

\section{A. Motivation}

The amount of data flow in the data plane is the most important issue in the field of traffic management and load balance in SDN networks. As the number of sensing devices that communicate with the switches in data plane is increased, the traffic load in the queuing buffer of the SDN-IoT gateway will also be increased. Also, as the number of switches in an SDN increases, the performance of the centralized controller in its control plane will fail to process all the requests coming from the switches. The use of artificial intelligent networks and machine learning with SDN has received increasingly marked interest in recent years. [7] gives an overview of machine learning algorithms that have been applied in the realm of SDN. Combined with SDN, Artificial Intelligence (AI) can provide solutions to network problems based on classification and estimation techniques [8]. Intelligent traffic prediction is an important issue in SDN-IoT. Deep learning based on an artificial neural network (ANN) has demonstrated its proficiency in traffic management, load balance and routing in SDN networks [9]-[14]. One crucial requirement for improving network performance is optimizing the routing process of SDN, while maintaining the QoS [14]. The traditional SDN implementation based on a logically centralized controller has several constraints, including poor scalability and unreliable performance. With the fast growth of Internet flow and scale, this means that network sensor devices are widely spread, but the network range that a single controller can support is limited. In order to address the problem of low network performance and single point malfunction caused by exceeding traffic for a single controller, multiple controllers are usually implemented in the network to deliver distributed control management. With this arrangement, the control plane is split into several sub realms, with each controller only needing to manage the switches in its own. This can alleviate 
the deficiencies of the control plane in terms of reliability, scalability and versatility [15].

The design of an intelligent controller based on AI is the main topic in this paper. However, it is deemed appropriate to choose an algorithm that is more biologically realistic than an ANN. Spiking Neural Networks (SNNs) the "third generation of ANNs" are so and arguably the only viable option, if the aim is to gain clear insights into how the brain computes. Moreover, SNNs are more hardware friendly and energy-effective than ANNs [16]. SNNs are dynamic systems, with time being a more important factor than for conventional feedforward ANNs [17].

\section{B. Contributions}

This paper introduces a Partial Recurrent Spiking Neural Network (PRSNN) as a congestion controller in the proposed model. The PRSNN is a type of SNN with partial feedback in the hidden layer. Also, another controller based on ANN is introduced to manage the sensors in the spike ISDN-IoT network.

The main contributions of this paper can be summarized as follows

1.We propose a spike ISDN-IoT model with two intelligent controllers based on AI, both of which are placed in the SDN control plane. One of them, which is based on PRSNN, estimates the amount of packet flow in the network, whilst the other, which is based on an ANN controller, selects and manages the cluster head of the sensors in the sensing area.

2. We propose an intelligent queuing model to estimate the capacity of the buffer size in the spike ISDN-IoT network based on a PRSNN controller.

3. We propose a modified training algorithm PRSNN to update its weights, the delay and the threshold values.

The remainder of this paper is organized as follows. Section II reviews related works, section III presents the proposed system model with the network architecture and section IV presents the modified training algorithm. Then, in section V, the evaluation setup is presented and in section VI the results are shown, with the QoS improvements being discussed. Finally, in section VII the conclusion to the paper is provided.

\section{RELATED RESEARCH WORK}

This section introduces the most recent research relating to the use of deep learning in traffic management and load balance applications in SDN networks. Mao et al. [14] proposed a non-supervised deep learning convolutional neural network (CNN) based routing methodology for a Software Defined Wireless Network, which can control the traffic of the network better than conventional routing protocols, with higher service quality.Tang et al. [9] proposed two deeplearning CNNs based on intelligent partial overlapping channel assignment to route traffic in a wireless SDN-IoT network, which improves the performance of the network. they utilized deep learning to predict the future traffic loads of switches.

Tang et al. [12] proposed a deep learning CNN based traffic load prediction algorithm for predicting traffic load at the next time interval and preventing congestion in an
SDN-IoT network, which significantly outperforms the conventional method. Mao et al. [13] proposed intelligent routing based on a real-time deep learning strategy for a CNN in an SDN communication system. Yu et al. [10] suggested a deep reinforcement learning mechanism for an SDN to optimize the routing of the sensing area, which provides good convergence and effective routing services. Lin and Tsai [18] proposed a controller system for enhancing network scalability and reducing computation delay in SDNs, whilst meeting QoS requirements based on hierarchical edge-cloud SDN (HECSDN). Xu et al. [19] showed that multiple distributed controllers can be used in SDNs to improve scalability and reliability, where each manages one static partition of the network. The SDSense is a novel architecture proposed in [20], which entails an SDN based WSN design, where software enabled sensors are dynamically reconfigured to adapt to current network conditions, which significantly improves the network performance. Misra et al. [21] proposed a situationaware protocol switching scheme for software defined wireless sensor networks to support application in real-time.They showed that their protocol is capable of enhancing the network performance. Dias et al. [22] designed and implemented a scalable system architecture that integrates a WSN into IoT. Priority-based virtual machine allocation and a network traffic management scheme with bandwidth allocation along with a dynamic flow pathing mechanism was proposed by Son and Buyya [23]. Al-Shammari et al. [24] proposed a traffic flow management policy to allocated and organized traffic flow network resources.

AI has become a very important issue and researchers have been devising procedures for improving this area in the field of training algorithms, where SNNs are proving to be remarkably effective. There are many algorithms that have been proposed and implemented for training an SNN [17], [25]-[29].

Different from the reviewed literature, this paper implements two intelligent controllers in the spike ISDN-IoT control plane based on AI. Also, we present a modified training algorithm to enhance the controllability of a spike ISDN-IoT network. The modification of the training algorithm is based on the spike back propagation (SBP) [25], [29]. Our proposed algorithm introduces a further training mechanism to prevent the occurrence of unwanted spikes that may lead to errors in the predicted level of traffic. In an attempt to enhance the efficiency of the proposed model (spike ISDN-IoT), we compare it with the CNN.

\section{SYSTEM MODEL}

Fig. 1. shows the proposed model that is introduced in this paper. The developments that have occurred in the science of networks, communications and artificial intelligence have prompted the use of these technologies in various aspects of life. Our focus is on the application of the proposed model in the field of health, especially in hospitals in Iraq. The model consists of a sensing plane, control plane and application plane of an spike ISDN-IoT network. 


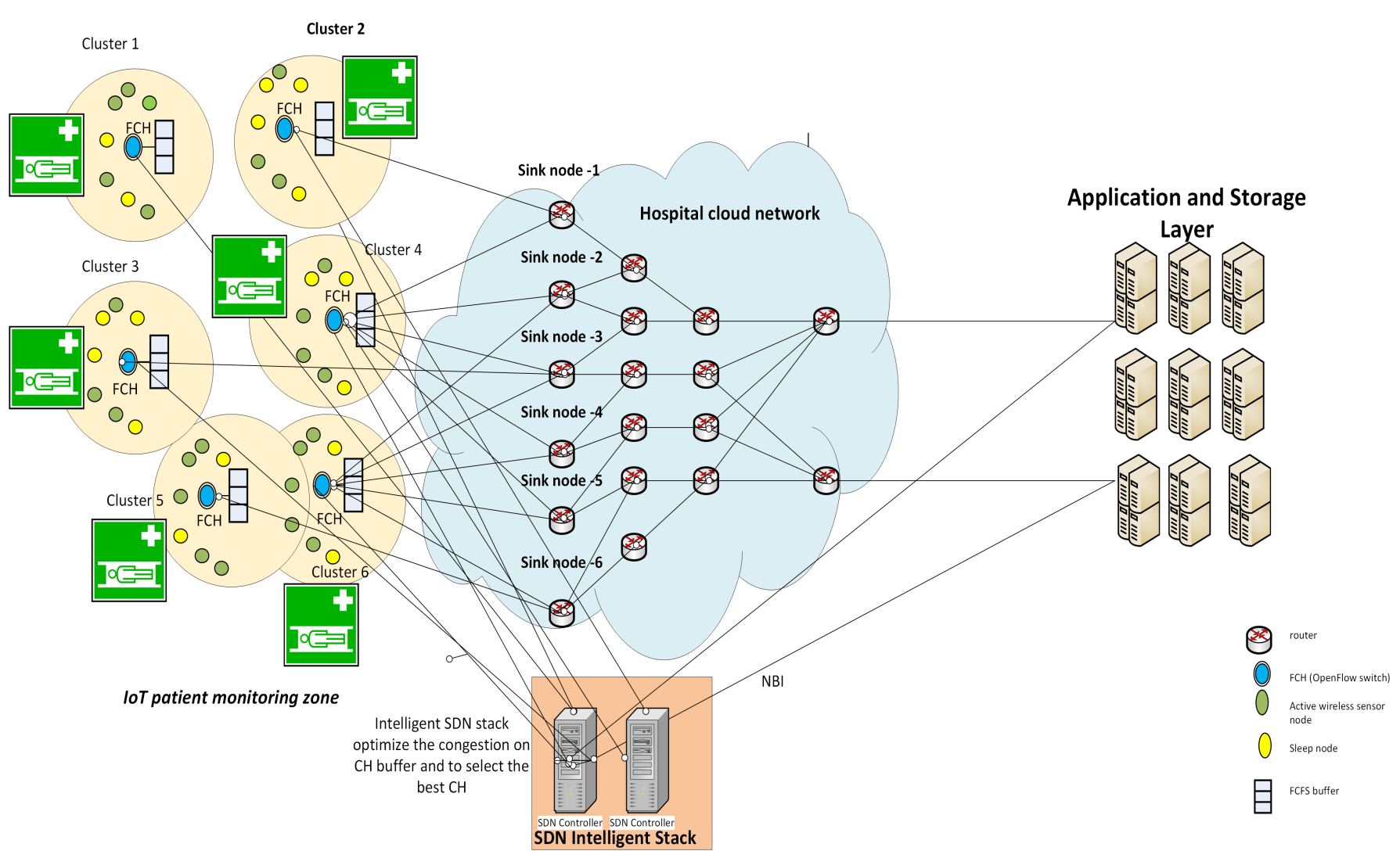

Fig. 1. Proposed Spike ISDN-IoT Network.

\section{A. Sensing plane}

The proposed model consists of an IoT patient monitoring zone, which is defined as the number of wireless sensing nodes in the sensing area classified according to their activity into three types, as: Forwarding Cluster Head (FCH), which we refer to as the OpenFlow switch; active node; and sleep node. Active member nodes transmit their data to an $\mathrm{FCH}$ and in turn, it forwards aggregated data to the sink node as a GATEWAY (GW), the internal components of which are shown in Fig 2 In practice, the GW connects the WSN using a point-to-point connection over the Internet. That is, it can connect to the Internet via local routers with firewalls. [22].In this paper, we propose an intelligent SDN architecture for routing and traffic management of patient sensor data. The packet flow that arrives from the buffer of the FCHs with a number of active sensors is destined to the hospital cloud network, as shown in Fig. 1. The FCH approach has two phases: setup and steady-state. In the setup phase, where the FCHs are chosen, each sensor node belongs to its $\mathrm{FCH}$ and a cluster is formed, every sensor node that is not an $\mathrm{FCH}$ determining its neighbors and its distance. Secondly, during the steady-state phase, every active sensor begins to send data to its FCH. The FCH approach takes into account some basic factors: residual energy of the sensor nodes, their density and the residual capacity of the buffer size. This is explained in the following equation.

$$
I R_{N}=f\left(\left\{E N_{N} \times \alpha_{N} \times d_{N} \quad \text { if } \quad d_{N} \geq d_{t h}\right\}\right)
$$

where, $I R_{N}, E N_{N}$ and $d_{N}$ represent the weight, the residual energy and the density of the sensor $N$ sequentially. $f(\cdot)$ is a nonlinear function which represents the performance of the ANN reactive controller, and $d_{t h}$ is the minimum density threshold.The term density of one node is the amount of aggregated neighboring nodes in a place in range r. $\alpha_{N}$ being the factor of flow buffer size capacity for every sensor as in the following equation:

$$
\alpha_{N}=\frac{\alpha_{\max }^{N}}{\text { no. of alive sensor nodes in range } r} .
$$

where, $\alpha_{M A X}^{N}$ is the maximum capacity of flow buffer size in the sensor. Each node manages itself in terms of determining whether to be active and be able to transmit its data or remain in sleep mode. To avoid congestion in the FCHs' flow buffer that might not have enough capacity to accommodate the sensory-data, the approach has the capability of making the number of active sensors coordinate with their FCHs' flow buffer size. The number of active nodes $S_{A}$ is determined as in the following equation:

$$
S_{A}=\frac{\text { flow table size of } F C H}{\text { total rate of sensor }}
$$

The proposed FCH approach is used to improve the QoS by reducing packet flow loss and reducing overflow on the $\mathrm{FCH}$ flow buffer. The sensor nodes can generate data packets and forwarding data as OpenFlow switches do. 


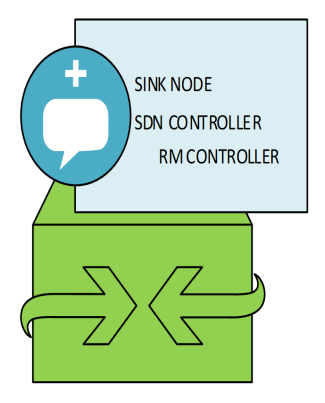

GATEWAY

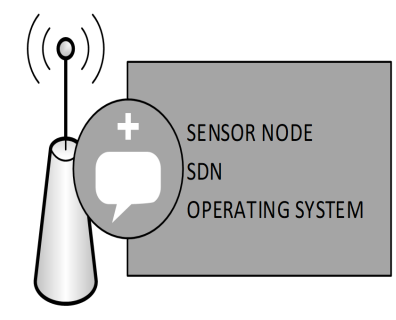

Sensor node
Fig. 2. The internal structure of gateway and sensor node.

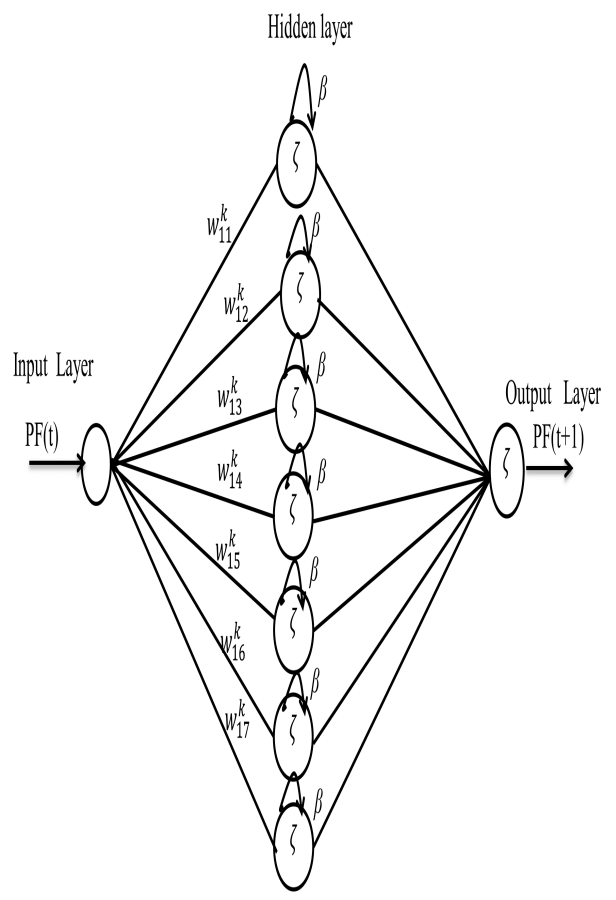

Fig. 3. Structure of the partial recurrent spike neural network.

\section{B. control plane}

Consider that spike ISDN-IoT is contracted in a homogeneous network, as shown in Fig. 1. consisting of a number of sensors used to sense data from different devices with different types of traffic. The periodic data are collected from a sensor, e.g., the temperature of a patient or blood pressure. In our case, the sensors can collect patient data dynamically to stimulate preventive care, diagnostics etc. and to measure treatment results. The hospital cloud network in Fig. 1 consists of a number of routers, the number depending on the number of considered switches. Each router has its First-come First Served (FCFS) buffer with a predefined capacity. OpenFlow

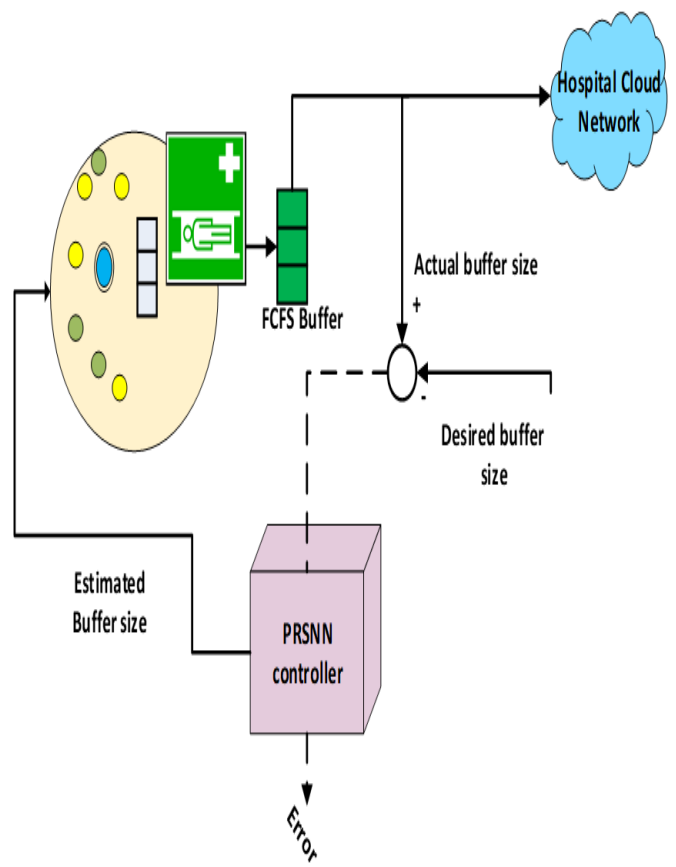

Fig. 4. The proposed queuing model.

was designed as one of the first SDN standards.It basically defines the communication protocol in SDN environments as it enables the SDN controller to directly combine with its data plane.The communication delay between the data plane and control plane is neglected as it is negligible compared to the distance between data plane and cloud.

\section{The Intelligent SDN Stack}

SDN technology can work with WSN to verify the activation of sensor nodes in real-time to meet application requirements [21]. The intelligent controllers are the brain of the SDN control layer, which manage the traffic flow of spike ISDN-IoT. We propose an SDN intelligent stack that has two intelligent controllers. These controllers are described as follows:

1) PRSNN Congestion Controller: The structure of PRSNN consists of one input node, a hidden layer with a number of neurons with self-feedback and one output node, as shown in Fig 3 The presence of many hidden layers decreases the speed of the training process and increases network complexity. The PRSNN controls and estimates the packet flow $(p f)$ for the next round in order to reduce the congestion that could occur in the network.

Fig 4 shows the proposed queuing model, where error $(\mathrm{t})$ is the difference between the desired and actual occupancy 


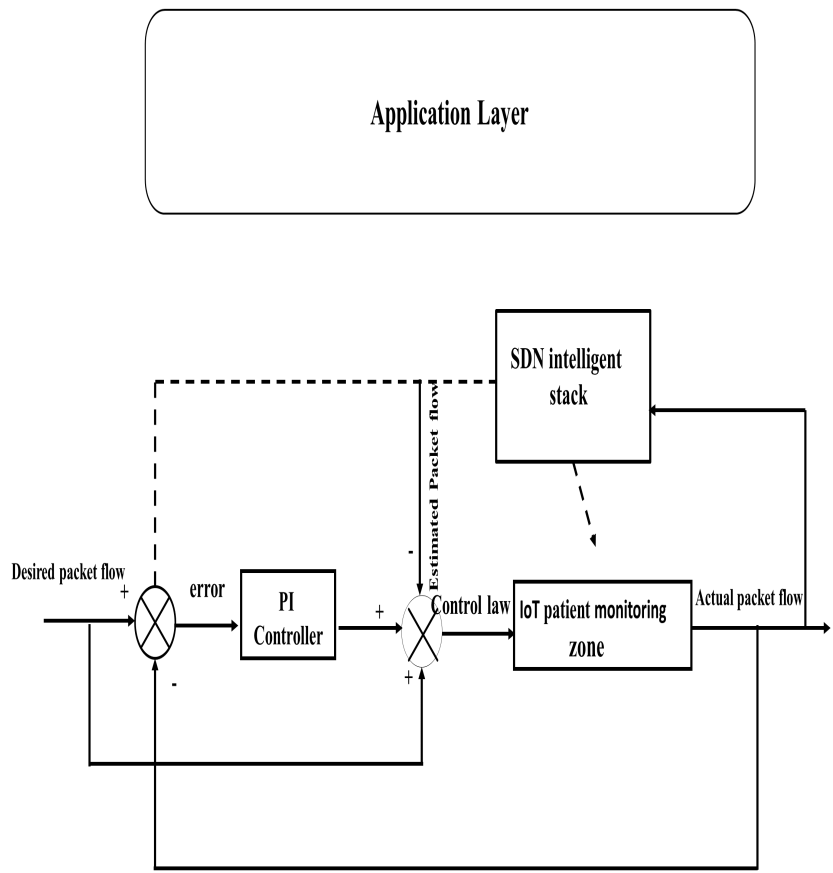

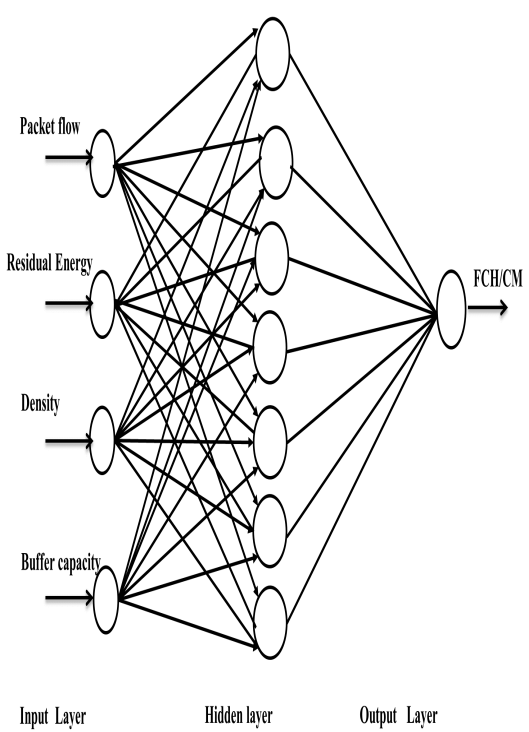

Fig. 6. The structure of the artificial neural network selection process .

PRSNN in Fig 5 trains on-line to estimate the packet flow. the ${ }_{303}$ minimum rate $b_{N}$ at the sensor $\mathrm{N}$, is defined as:

$$
b_{N}=Q_{M} \log \left(R_{M}\right)
$$

where, $Q_{M}$ is the size of the queue (buffer) of the (M) FCH node with the corresponding rate $R_{M}$. The optimization issue assigns link bandwidth in such a way that the overall ISDNIoT network utilization $N_{U}$ is maximized as in the following formula:

$$
N_{U}=\operatorname{maximize} \sum_{M} Q_{M} \log \left(R_{M}\right)
$$

$$
p f(k+1)=\operatorname{sat}[f f(p f(k)+T u(k)]
$$

Where, $p f(k)$ is the packet flow at time $k, T$ is the sampling period, $u(k)$ is the control law signal and sat $[\cdot]$ is the saturation function. The nonlinear function $f f(\cdot)$ represents the actual packet flow, which is considered as being unknown. The $f f(\cdot)$ is also a function of buffer size, traffic input and available service capacity at the given sensor nodes. The packet flow rate input controller is calculated as:

$$
u(k)=\frac{1}{T}\left(p f d-\hat{f}(p f(k))+k_{v} e(k)\right)
$$

where, $k_{v}$ is the coefficient of the proportional integral controller (PI) used here to increase the accuracy and to eliminate the steady state error as well as keeping the network stable throughout the training process, while $\hat{f}(p f(k))$ is the estimated packet flow and the $p f d$ is the desired packet flow.
2) The ANN Controller: The other intelligent controller is based on an ANN (FeedForward Neural Network with one hidden layer), as shown in Fig 6 . We are proposing it being used to select the best FCH OpenFlow to carry traffic.The IoT patient monitoring zone is managed based on an ANN, taking the factors described in section III (A) as input to it, while its output is the logical value, where logic 1 is defined as an $\mathrm{FCH}$ and logic 0 are cluster members (CM).The back-propagation training algorithm is used to update the weights in an on-line manner.

\section{MODIFIED TRAINING ALGORITHM}

In this section, the modified training algorithm used to train the PRSNN controller is explained. The proposed algorithm is based on the scope of the negative gradient descent method for minimizing the difference between the desired and actual packet flow. The parameters that are trained in the proposed 
algorithm are the: weights, sub-connection or synaptic delays, and threshold).

The internal connection single synaptic of PRSNN is shown in Fig. 7. A and the broken line portion of single synaptic terminal in Fig $7 \mathrm{~B}$. represents two neurons with a time delayed synaptic link. The neuron $i$ in the Fig. 7 $\mathrm{B}$. is not allowed to spike anymore through the remaining period of time interval $\mathrm{T}$, when the threshold value $\theta$ has been exceeded at a particular instant $t_{i}$ and it will be reset in the next, $t_{i}+d^{k}$. Every single connection among the layers in PRSNN is composed of a group with the same number of synaptic terminals. Every sub-connection is related with a different weight and delay, as it is clear in Fig.74. The difference between the time of the postsynaptic potential and the firing of presynaptic neurons $i$ is defined as the delay of the synaptic terminals. The time of postsynaptic potential begins to rise, as seen in Fig $7 \mathrm{~B}$, and there is a synapse sequence in the connection.The weight of each synapse has an effect on the spike-response function $\zeta$. The input of PRSNN is assigned to the packet flow accumulation rate $p f(t)$, i.e., the number of flow packets arriving at the SDN controller from the network. The number of sub-connections or synapses in the relationship between the input and hidden layers as well as between the hidden and output layers is updated. This number is typically chosen empirically at the initial stage.To start with, the weights are initiated randomly between [-0.5-0.5] and then, after implementing rounds of training, the weight values are tuned more efficiently with the adaptive learning rate $\eta$.

The desired and the actual packet flows are firstly encoding into spike times based on following equation:

$$
t_{h}^{f}=t_{\max }-\left\lfloor\frac{t_{\min }\left(p f(t)-p f_{\min }\right)\left(t_{\max }-t_{\min }\right)}{\left(p f_{\max }-p f_{\min }\right)}\right\rceil .
$$

where, $p f_{\max }$ and $p f_{\min }$ represent the maximum and minimum real flow, whilst $t_{\max }$ and $t_{\min }$ are the maximum and minimum interval time, respectively. The function \lfloor\rceil is a round function.

The flow packet decoding equation is described by:

$$
p f\left(t_{j}\right)=\frac{\left(t_{\max }-t_{j}-t_{\min }\right)\left(p f_{\max }-p f_{\min }\right)}{\left(t_{\max }-t_{\min }\right)}+
$$

There are two modes for the training algorithm. The first is called the feed-forward mode, where each neuron spikes at each time interval $\mathrm{T}$ only once at most and this happens when the value of threshold $\theta$ is exceeded by the membrane potential $m$. The feed-forward mode always begins from hidden layer $I$ with neuron $(i)$ being continuously examined to see whether it is spiked or not. The algorithm uses the next neuron $(i+1)$ when the neuron $(i)$ is spiked. The membrane potential $m_{i}(t)$ is calculated by the training algorithm according to $(10)$ based on input spikes $t_{h}^{f}$ of neuron $h$ at the input layer.

$$
\begin{array}{r}
m_{i}(t)=\sum_{h=1}^{N H} \sum_{k=1}^{D} w_{h i}^{k} \zeta\left(t-t_{h}^{f}-d^{k}\right) \\
+\beta * \sum_{h=1}^{N H} \sum_{k=1}^{D} w_{h i}^{k} * p f_{h i}^{k}(t-1) .
\end{array}
$$

where, $\beta$ is a self-feedback in PRSNN structure and it is a constant value between $(0-1)$. The term $p f_{h i}^{k}(t-1)$ represents the previous packet flow as the input to the PRSNN. The activation function $\zeta\left(t-t_{h}^{f}-d^{k}\right)$ is defined as:

$$
\zeta\left(t-t_{h}^{f}-d^{k}\right)=-\sigma * \exp \frac{-\left(t-t_{h}^{f}-d^{k}\right)}{\tau} .
$$

The same process will be repeated in the output layer $J$ by the algorithm when the second layer's neurons have finished, which is when the back-propagation phase begins.

The synapse weights of connection are updated when the feed-forward mode has finished. Opposite to feed-forward, back-propagation begins from the output layer and returns back to the hidden layer. For simplicity, we defined the function $\zeta\left(t-t_{h}^{f}-d^{k}\right)$ as $y_{h}^{k}$ and $\zeta\left(t-t_{i}^{f}-d^{k}\right)$ as $y_{i}^{k}$.

$$
E=\left(T_{j}-t_{j}^{f}\right)
$$

where, $E$ represents the error between the desired spike time and actual spike time of the neuron. The synapses of the hidden layer and output layer will be updated according to (13) 18 .

$$
w_{i j}^{k}(t+1)=w_{i j}^{k}(t)-\Delta w_{i j}^{k}(t) .
$$

where,

$$
\Delta w_{i j}^{k}(t)=\eta \cdot \delta_{j} \cdot y_{h}^{k}
$$

$$
\begin{gathered}
\delta_{j}=\frac{E}{\sum_{(i=1)}^{I n} \sum_{(k=1)}^{D} w_{i j}^{k} \frac{\partial y_{i}^{k}}{\partial t}} . \\
\delta_{i}=\frac{\sum_{(i=1)}^{(I n)} \delta_{j} \sum_{(k=1)}^{D} w_{i j}^{k} \frac{\partial y_{i}^{k}}{\partial t}}{\sum_{(i=1)}^{H n} \sum_{(k=1)}^{D} w_{h i}^{k} \frac{\partial y_{h}^{k}}{\partial t}} . \\
w_{h i}^{k}(t+1)=w_{h i}^{k}(t)-\Delta w_{h i}^{k}(t) .
\end{gathered}
$$

where,

$$
\Delta w_{h i}^{k}(t)=\eta \cdot \delta_{i} \cdot y_{i}^{k} .
$$

All the symbols in the above equations are described in Table I. The update of the synaptic delay and neuron thresholds is explained in the following equations:

$$
\begin{aligned}
& \Delta_{h i}^{k}=-\left.\rho_{d} \sum_{(i=1)}^{(N I)} \frac{\partial E}{\partial t_{j}^{f}} \frac{\partial t_{j}^{f}}{\partial y_{h}^{k}(t)} \frac{\partial y_{h}^{k}(t)}{\partial d_{h i}^{k}}\right|_{\left(t=T_{j}\right)} . \\
& \Delta \theta_{j}=-\left.\rho_{\theta} \sum_{(i=1)}^{(N I)} \frac{\partial E}{\partial t_{j}^{f}} \frac{\partial t_{j}^{f}}{\partial y_{h}^{k}(t)} \frac{\partial y_{h}^{k}(t)}{\partial \theta_{j}}\right|_{\left(t=T_{j}\right)} .
\end{aligned}
$$


TABLE I

Parameters of the partial recurrent spike neural network training algorithm

\begin{tabular}{|c|c|}
\hline Symbol & Meaning \\
\hline$\sigma$ & Constant of the activation function \\
\hline$\eta$ & Learning rate \\
\hline$\theta$ & The threshold value \\
\hline$\rho_{d}$ & Learning rate of the synaptic delay \\
\hline$\rho_{\theta}$ & Learning rate of the synaptic thresholds \\
\hline$\tau$ & The time constant \\
\hline$\delta$ & The delta function \\
\hline$d^{k}$ & delay of the connection \\
\hline$m_{i}$ & Membrane potential of neuron $i$ at the hidden layer \\
\hline$m_{j}$ & Membrane potential of neuron $\mathrm{j}$ at the output layer \\
\hline$w_{h i}^{k}$ & Sub-connection weight between the input and hidden layers \\
\hline$w_{i j}^{k}$ & Sub-connection weight between the hidden and output layers \\
\hline$\Delta t$ & Step time \\
\hline$D$ & Number of delayed synapses per connection \\
\hline $\bar{H}$ & Input layer \\
\hline$I$ & Hidden layer \\
\hline$J$ & Output layer \\
\hline$T_{j}$ & Desired spike time of the output neuron \\
\hline$t_{j}^{f}$ & actual spike time of output neuron \\
\hline $\mathrm{NH}$ & Number of neurons in the input layer \\
\hline$N I$ & Number of neurons in the hidden layer \\
\hline$y_{h}^{k}$ & The output of the hidden layer \\
\hline$y_{i}^{k}$ & The output of the output layer \\
\hline$T$ & Time interval \\
\hline Epoch max & Maximum number of epochs \\
\hline$h$ & Neuron sequence in the input layer \\
\hline$i$ & Neuron sequence in the hidden layer \\
\hline$j$ & Neuron sequence in the output layer \\
\hline
\end{tabular}

The parameters are updated in the training algorithm with the initial values are chosen by trial and error. PRSNN is adaptive according to the traffic dynamics and the data plane performance, such that the proactive controller keeps a balance between the buffer sizes and traffic flow of the network.PRSNN achieves both data plane efficiency (high traffic flow rate) and stability. The flow chart of the proposed model is shown in Fig. 8 and the training algorithm of PRSNN is shown in Figs. 9 and 10 .

We consider scenarios with $\mathrm{N}$ sensors that are placed in a random way in a sensing square area of (150X150) meters, with the transmission range of each sensor being fixed at $25 \mathrm{~m}$. We vary the number of sensors (80 and 120) to control the density of the network and the implementation for the area is shown in Fig 11 The sensors generate traffic at the beginning of each scheduling period.That is, they implement low to high flow and then, this traffic is routed to the FCH. The PRSNN controller contributes to minimizing the congestion level. That is, the FCHs are classified as congestion, if this percentage exceeds a threshold level. In this paper, the threshold level is set at $90 \%$ of the queue buffer size and it is selected based on experiential evaluation.

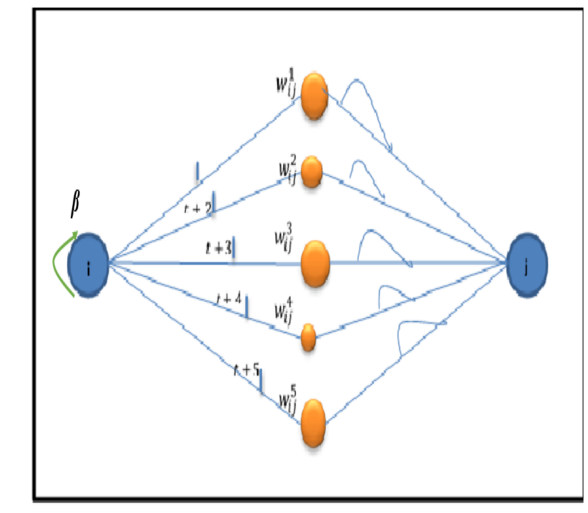

A

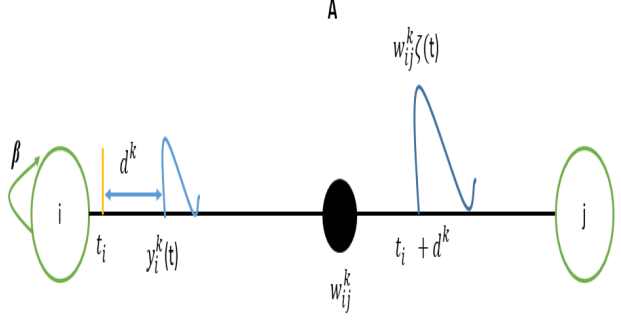

B

Fig. 7. A: Internal connection single synaptic of a partial recurrent spike neural network. B: Single synaptic terminal.

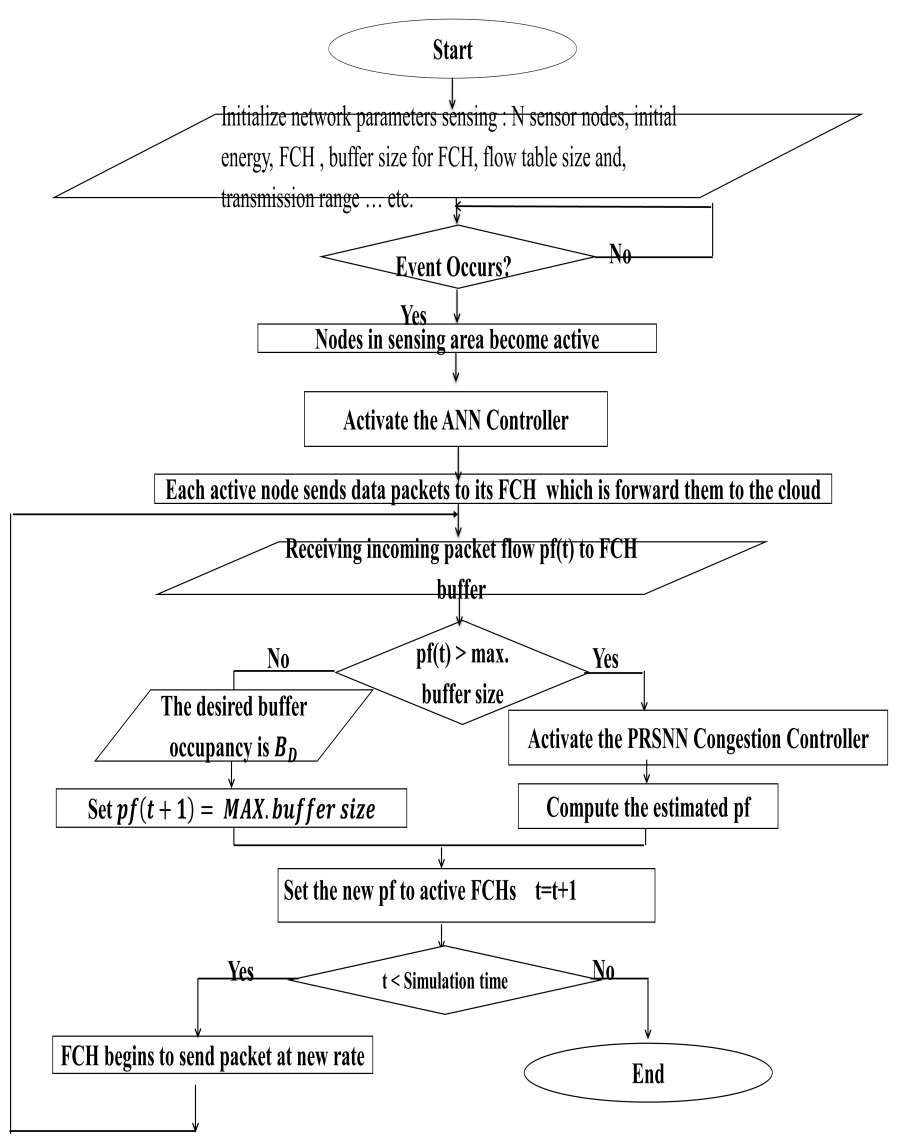

Fig. 8. Flowchart of the proposed model. 


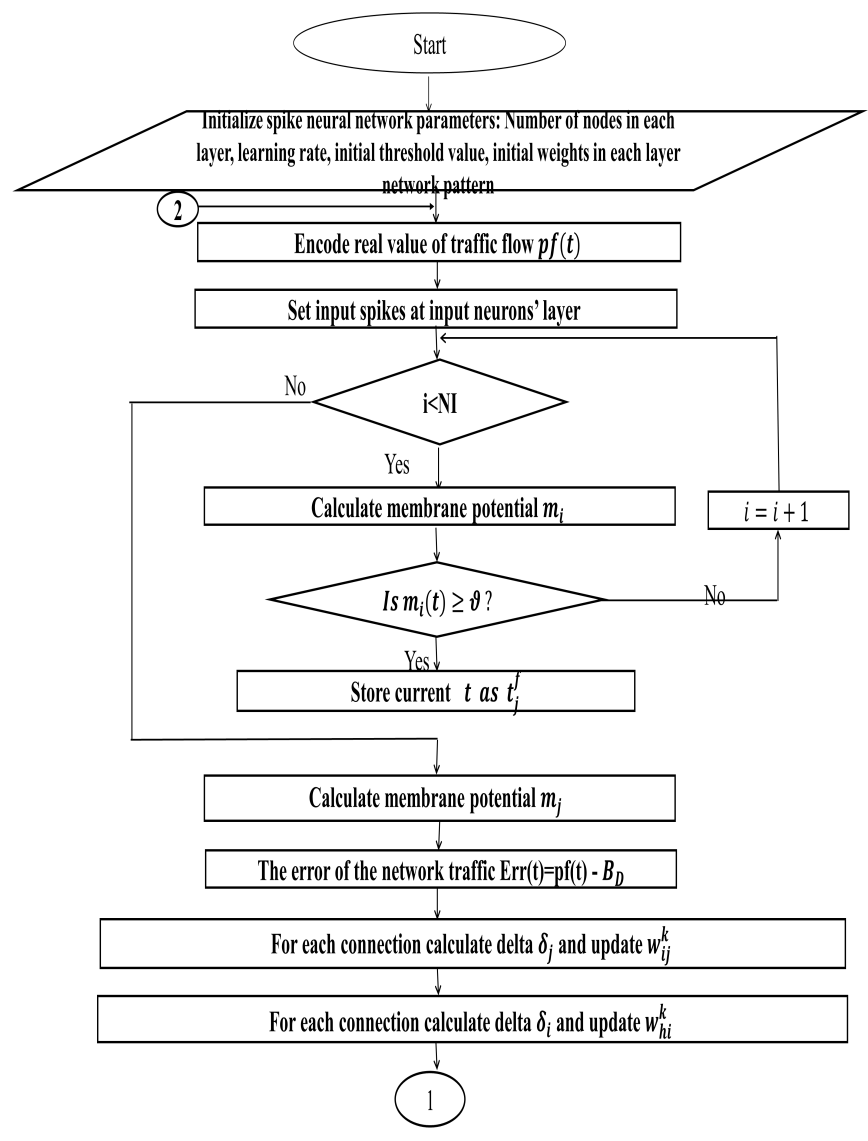

Fig. 9. The proposed training algorithm.

TABLE II

PARAMETERS OF THE SIMULATION

\begin{tabular}{|c|c|} 
& \\
\hline Coverage area & 150 meters X 150 meters \\
\hline Number of nodes & 80,120 \\
\hline Buffer size of FCH & 250 PACKETS \\
\hline Buffer size of each sensor node & $50-100$ packets \\
\hline Data packet size & 800 byte \\
\hline Simulation time & $250 \mathrm{msec}$ \\
\hline Data packet generating for each node & $5($ packet $/ \mathrm{msec})$. \\
\hline
\end{tabular}

The simulation is run with the parameters described in Table
II and with the Python programming language and Mininet simulator.

The following assumptions are applied for the network:

1. All stationary active sensor nodes generate static flow per unit of time;

2. There are two activities for the sensor node, the first being to generate flow traffic and the second is forwarding it to the $\mathrm{FCH}$;

3. The connection between the cloud, $\mathrm{FCH}$ and its member nodes comprises bidirectional single hop wireless links with an OpenFlow SDN switch;

4. Sensor nodes can verify their mode according to the FCH buffer capacity and its density;

5.The amount of flow (traffic generated) sent by the sensor node must be within the capacity of the channel of the

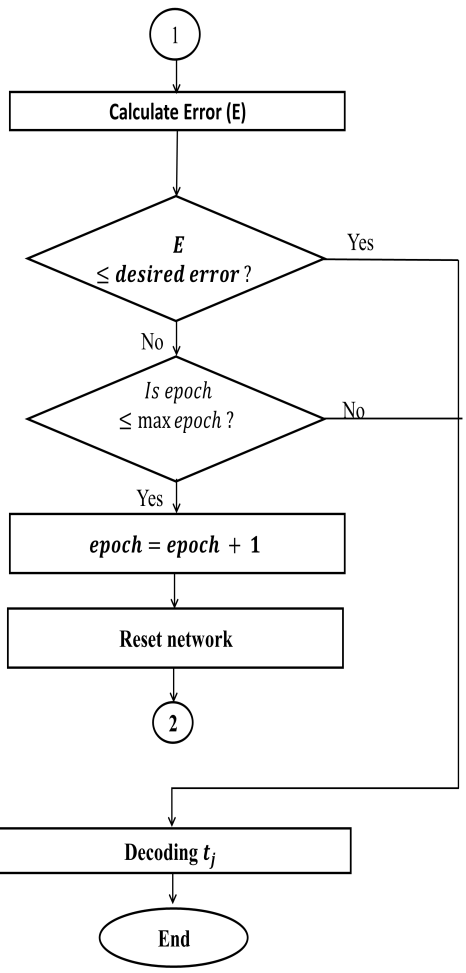

Fig. 10. Continue:The proposed training algorithm.

network.

To show the efficiency of the proposed model, a comparison is made between the it and that with a controller based on CNN. Fig. 12 shows the structure of CNN for a controller with one convolutional layer, a ReLU layer, and a fully connected layer used for estimated traffic in a spike ISDN-IoT network. The reason behind choosing CNN to compare with it, is that, it is more efficient than the traditional neural network, as explained in section II on related work.

The input of the CNN will be the features of the traffic flows, including the packet generation rate of every FCH,lengths of the packet queues in the buffers of the FCHs. The output is collected as two binary values, which when set at $(1,0)$ shows that the path mixture will lead to congestion and otherwise (i.e., 0,1), it will not. Clearly, the path mixtures that will not lead to congestion will be chosen. The CNNs will be periodically updated, while they are being used to select the path mixture. Every FCH will keep listing its traffic flow and then send the data to the SDN controller. The controller uses the data for the purpose that the traffic patterns of all $\mathrm{FCH}$ will be arranged in a matrix and then used as the input of the CNNs to choose the path mixture for the next time interval.

Fig. 13 shows the minimization of error during the training process. It is clear from the Fig. 13 that PRSNN can reach to the error goal faster than CNN can. This is because not all the neurons will update their weights all the time, but just 


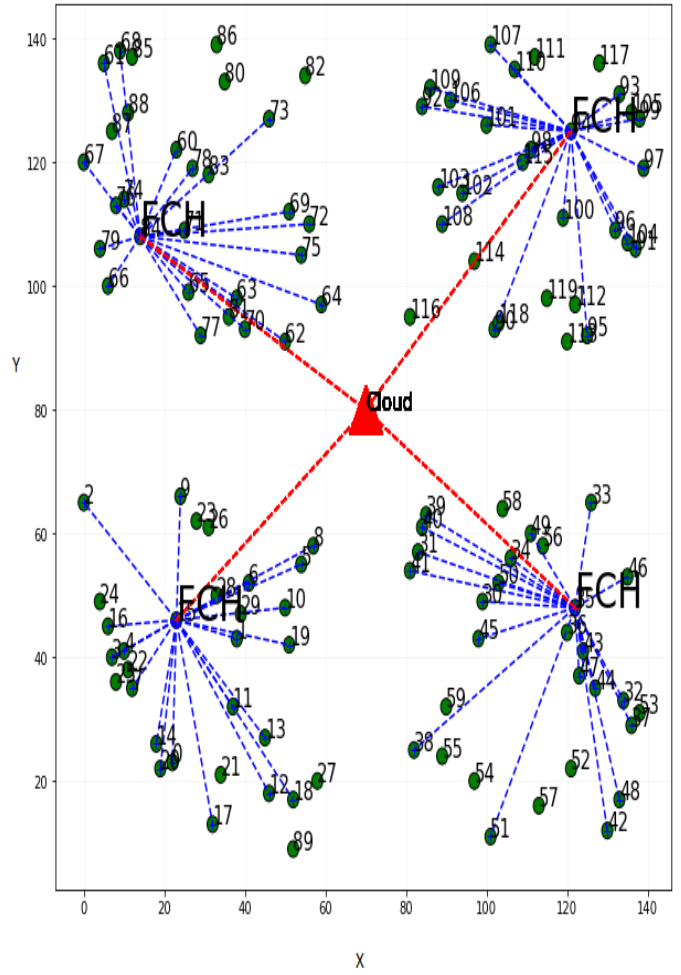

Fig. 11. The simulation area with 120 sensors nodes.

those which exceed the threshold value will be spike. So, the modified training algorithm which we propose to train PRSNN is more powerful than the back-propagation training algorithm used to train $\mathrm{CNN}$.

Fig 14 shows a comparison of the actual pf and estimated pf forwarded by the network and when the number of sensor nodes is 80 . It can be seen that the performance of the proposed congestion controller is better than $\mathrm{CNN}$, which is very clear when the network keeps its traffic with a buffer capacity size of FCH. In this simulation, we have four FCHs. When all are active, the network with the proposed model and CNN can operate in high traffic flow, thereby controlling the traffic in order to mitigate congestion at the buffer. The proposed model has a better ability at estimating the packet flow than with CNN. This is because the training algorithm can enhance the performance of PRSNN. It works with high capability of estimation of the ratio of packet flows. Fig. 15 illustrates the performance of the proposed model and CNN when the number of sensor nodes is increased to 120.Thus, the proposed model can work as accurately as CNN. In sum, the proposed congestion controller in the spike ISDN-IoT control plane is able to process all the requests coming from the switches even when the number is increased.

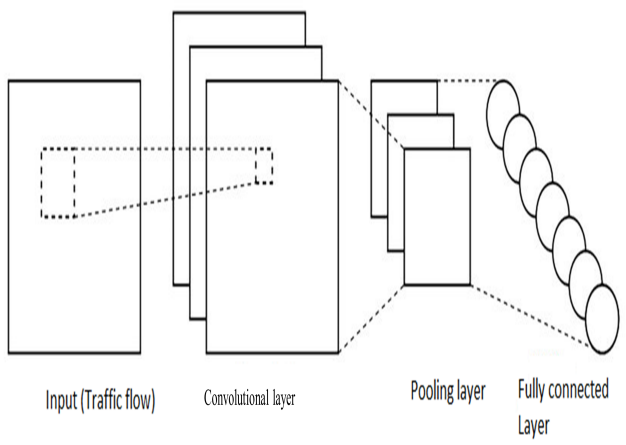

Fig. 12. The Convolutional Neural Network model.

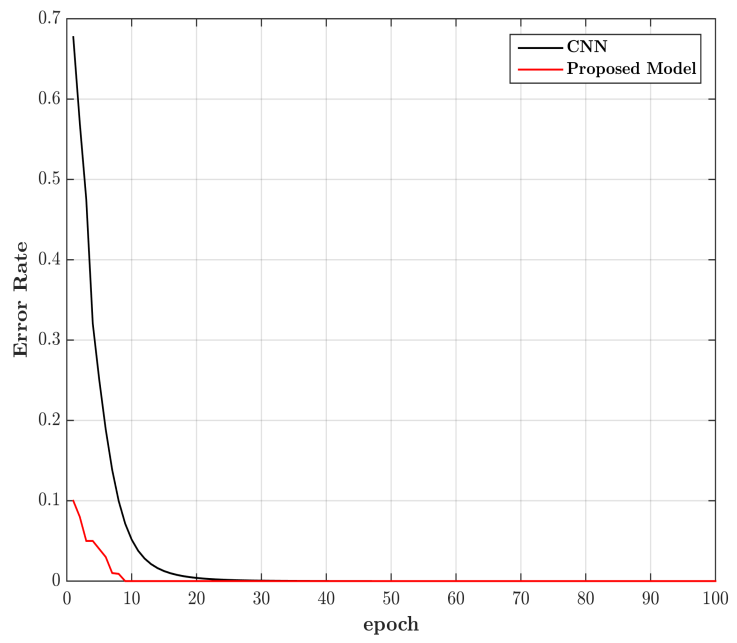

Fig. 13. The minimization of error during training.

\section{PERformance Metrics}

The performance of the proposed model, and CNN are explained with respect to QoS in terms of Packet Loss Ratio (PLR), Network Energy Consumption (NEC), Buffer Utilization Ratio (BUR), Network Throughput Ratio (NTR), and Network Life Time (NLT).

\section{A. Packet Loss Ratio (PLR)}

Fig. 16 presents the PLR in the spike ISDN-IoT network, when the proposed model is implemented. In Fig. 16, a comparison between the proposed model and CNN when the number of sensor nodes is 80 nodes is provided. We can observe from Fig. 16 that the PLR of the proposed model is 


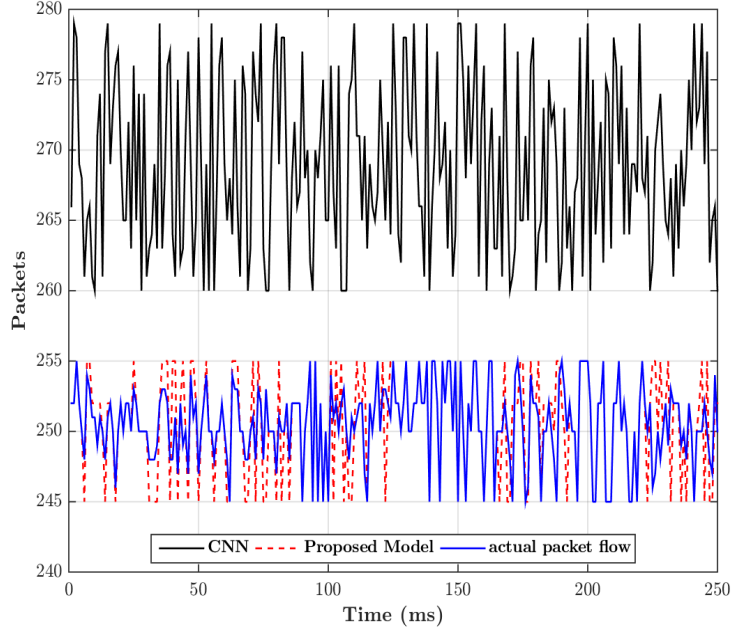

Fig. 14. Comparison of the estimated PF between the proposed model and $\mathrm{CNN}$ when the number of sensor nodes is 80

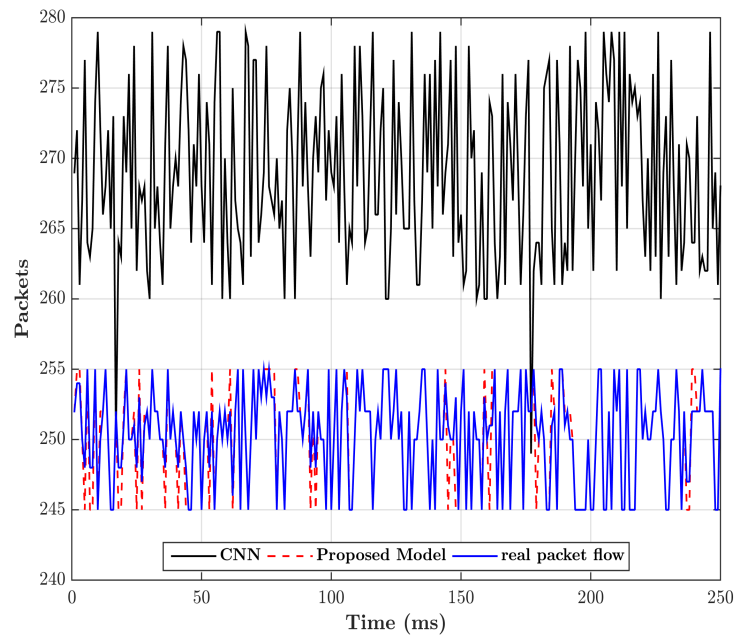

Fig. 15. Comparison of the estimated PF between the proposed model and CNN when the number of sensor nodes is 120

better than that for the $\mathrm{CNN}$, because the congestion controller is able to decrease the sending rate of the active clusters during the transmission process. It is also clear that whilst the CNN performs well, it is not as accurate as the proposed model. This means that, the proposed intelligent queuing model has good ability to estimate the capacity of the buffer size in the network and manage the queue of the packet flow accurately.

\section{B. Network Energy Consumption (NEC)}

Fig. 17 compares the energy consumption of $\mathrm{FCH}$ in the network for the proposed model and $\mathrm{CNN}$, with respect to time, when the number of sensor nodes is 80 . The result of the comparison demonstrates that the network energy consumption with the proposed congestion controller model is better than that with CNN. Thus,the proposed model aims at decreasing the energy consumed in the packets dropped due to overflowing the acceptable value. In the proposed training algorithm,

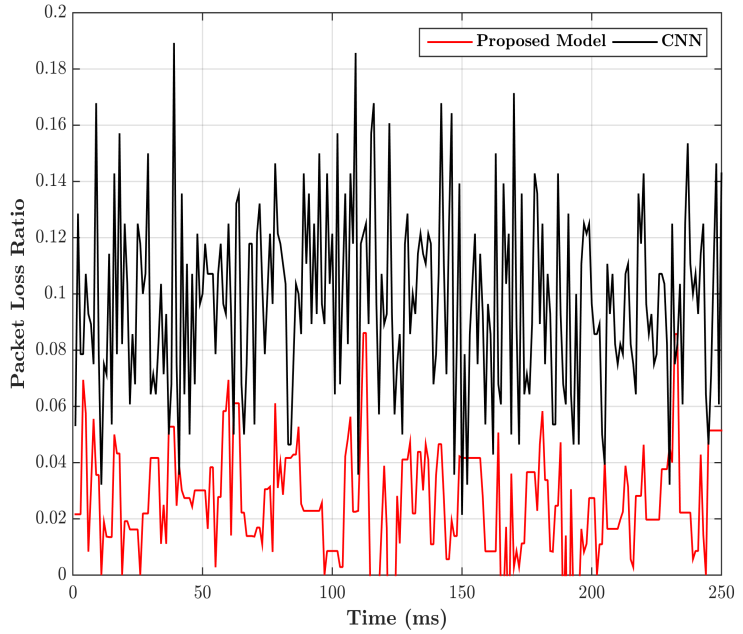

Fig. 16. Comparison of the packet loss ratio between the proposed model and $\mathrm{CNN}$ when the number of sensor nodes is 80 .

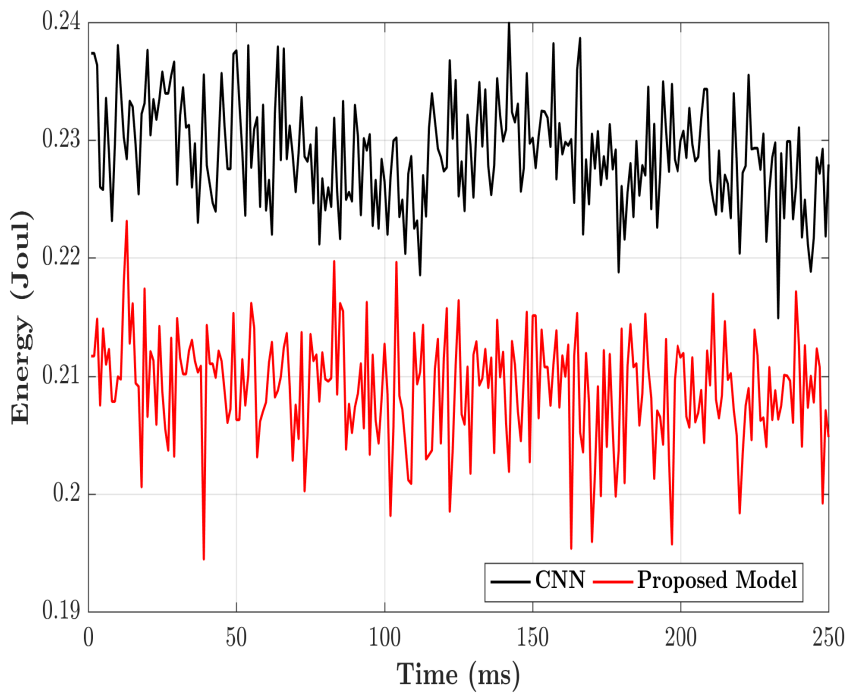

Fig. 17. Comparison of the network energy consumption between the proposed model and CNN when the number of sensor nodes is 80 .

not all the neurons are firing; just those that have reached threshold value. This means that the proposed model does not need as much time for training as with CNN. Also, separating the sensing area in the spike ISDN-IoT network into a number of FCHs, based on a ANN controller, provides the capability of minimizing the energy consumption of the whole network.

\section{Buffer Utilization Ratio (BUR)}

Fig 18 denotes the buffer utilization ratio of the network using the proposed model as compared with CNN, when the number of sensor nodes deployed in spike ISDN-IoT is 80. It is clear from Fig. 18 that the controlled network guarantees a good buffer utilization ratio. It is noted that the buffer utilization greatly exceeds that for CNN. Clearly, the proposed model performs well with high accuracy, much more so than with CNN.The idea behind using the PRSNN as congestion 


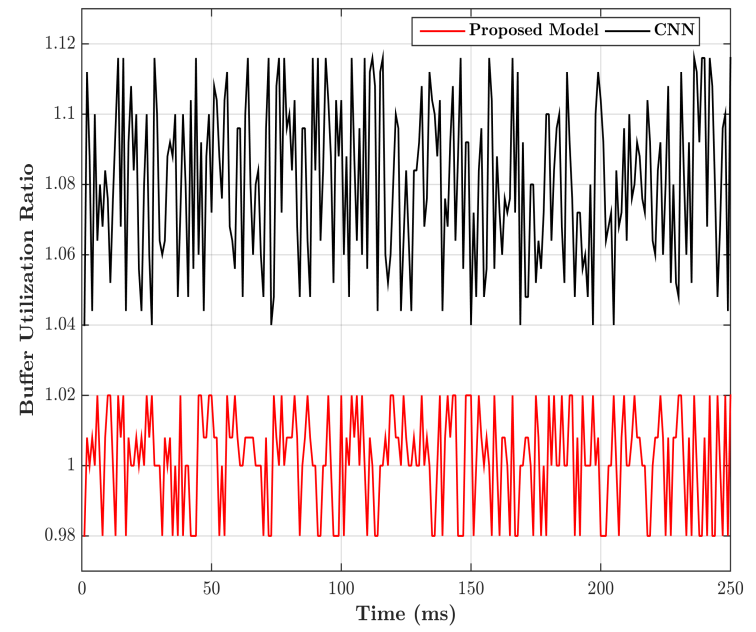

Fig. 18. The buffer utilization ratio when the number of sensor nodes is 80

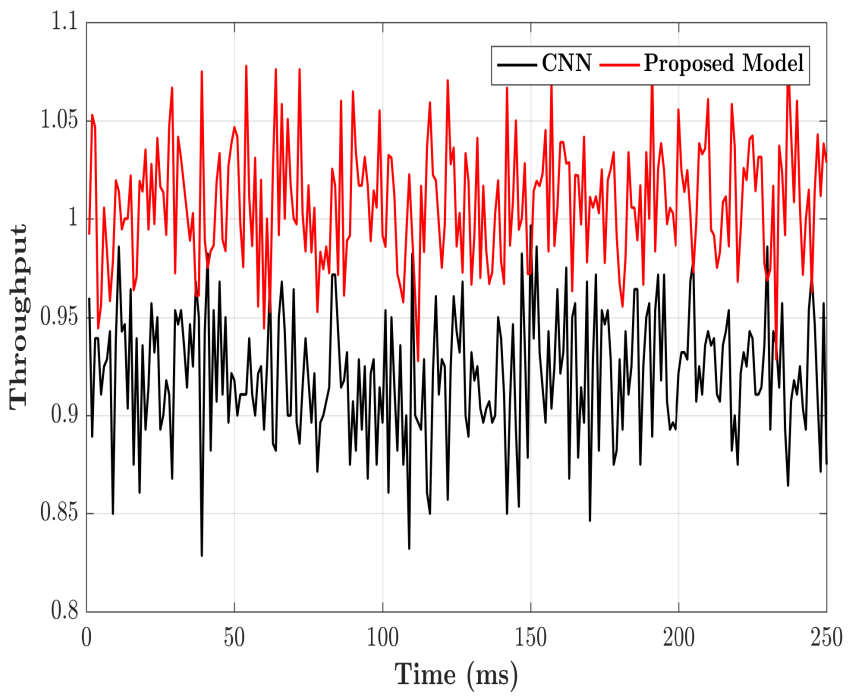

Fig. 19. The network throughput ratio when the number of sensor nodes is 80 controller is to increase the power of the network in estimating the packet flow. The strength of PRSNN is obtained from precise modeling of the synaptic interactions between the biological neurons, taking into account the time of spike firing. The computational power of PRSNN, thus outstrips that of CNN that uses threshold or sigmoidal activation functions. Furthermore, PRSNN has the potential for quick adaptation.

\section{Network Throughput Ratio (NTR)}

The NTR is defined as the proportion of the received packets by the gateway over the total number of packets generated by FCH during the simulation time. Fig. 19 display a comparison between the proposed model and the CNN, when numbers of sensor nodes is 80 . It is clear from the figure that the proposed model outperforms CNN, with higher throughput ratio.The spike ISDN-IoT network with the proposed model has success to keep the throughput ratio to $100 \%$ as compared with the percentage of CNN. In the proposed model, all the parameters (which have been described in section III) which are effective on the performance of the network, have been taken into consideration. The performance of the two controllers in our proposed model can efficiently manage the traffic load of the network. The spike ISDN-IoT network with the proposed

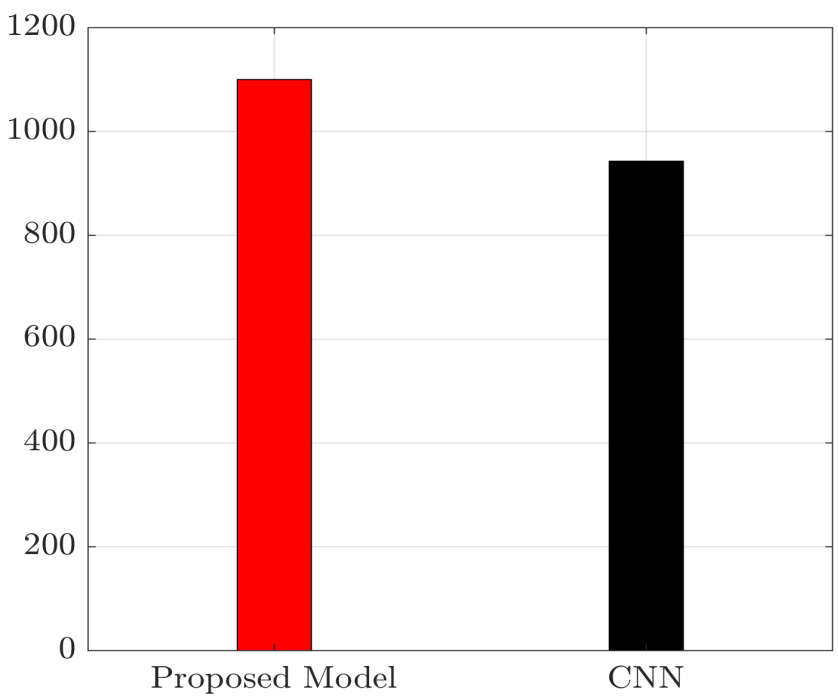

Fig. 20. The network lifetime

model has success to keep the throughput ratio to $100 \%$ as compared with the percentage of CNN. In the proposed model, all the parameters (which described in section III) which are effective on the performance of the network, have been taken into consideration. The performance of the two controllers in our proposed model are very helpful to manage the traffic load of the network.

\section{E. Network Lifetime (NLT)}

This refers to the time required to drain the energy of all the sensors nodes in the network. Fig. 20, shows a comparison of NLT when the proposed model and CNN are used. It is clear that the proposed model prolongs it more than CNN. The concept of FCH introduced in this paper with an ANN controller successfully increases the lifetime of the network, which means that the sensors of the sensing area can keep their energy for a longer time than with other methods, like CNN.

\section{CONCLUSION}

In this paper, we have proposed spike ISDN-IoT architecture for utilization in health care applications. We have proposed two intelligent controllers in the SDN intelligent stack, which has the capability of estimating the packet flow of the sensing area. One of the proposed controllers works proactively in a Partial Recurrent Spike Neural Network to estimate the packet flow of the sensing area.The other works as a reactive one based on an ANN, being tasked with selecting the cluster head and its members in the sensing area. The simulation results have proven that the QoS is enhanced in the spiked ISDN-IoT network. The ANN controller delivers the capability 
of selecting the cluster head and its members efficiently in sensing area, which is clearly shown in the results for QoS. The packet flow rate is estimated by the proposed model, which coordinates the available capacity of the buffer with a number of active sensor nodes in the network to prevent buffer overflow. Controlling the network by the proposed model has more accuracy than with $\mathrm{CNN}$, which is because of the spiking power of the proposed training algorithm.

\section{A. Acknowledgment}

This work is supported by Ministry of Higher Education and Scientific Research, University of Baghdad, Iraq.

\section{REFERENCES}

[1] S. Bera, S. Misra, S. K. Roy, and M. S. Obaidat, "Soft-wsn: Softwaredefined wsn management system for iot applications," IEEE Systems Journal, vol. 12, no. 3, pp. 2074-2081, 2016.

[2] A. Ghosh, D. Chakraborty, and A. Law, "Artificial intelligence in internet of things," CAAI Transactions on Intelligence Technology, vol. 3, no. 4, pp. 208-218, 2018.

[3] D. Kaur, G. S. Aujla, N. Kumar, A. Y. Zomaya, C. Perera, and R. Ranjan, "Tensor-based big data management scheme for dimensionality reduction problem in smart grid systems: Sdn perspective," IEEE Transactions on Knowledge and Data Engineering, vol. 30, no. 10, pp. 1985-1998, 2018.

[4] Z. Han, T. Lei, Z. Lu, X. Wen, W. Zheng, and L. Guo, "Artificial intelligence based handoff management for dense wlans: A deep reinforcement learning approach," IEEE Access, 2019.

[5] R. Thupae, B. Isong, N. Gasela, and A. M. Abu-Mahfouz, "Machine learning techniques for traffic identification and classifiacation in sdwsn: A survey," in IECON 2018-44th Annual Conference of the IEEE Industrial Electronics Society. IEEE, 2018, pp. 4645-4650.

[6] Q. Li, N. Huang, D. Wang, X. Li, Y. Jiang, and Z. Song, "Hqtimer: A hybrid $\{\mathrm{Q}\}$ - learning-based timeout mechanism in software-defined networks," IEEE Transactions on Network and Service Management, vol. 16, no. 1, pp. 153-166, 2019.

[7] J. Xie, F. R. Yu, T. Huang, R. Xie, J. Liu, C. Wang, and Y. Liu, "A survey of machine learning techniques applied to software defined networking (sdn): Research issues and challenges," IEEE Communications Surveys \& Tutorials, vol. 21, no. 1, pp. 393-430, 2018.

[8] A. Rego, A. Canovas, J. M. Jiménez, and J. Lloret, “An intelligent system for video surveillance in iot environments," IEEE Access, vol. 6, pp. $31580-31598,2018$.

[9] F. Tang, B. Mao, Z. M. Fadlullah, and N. Kato, "On a novel deeplearning-based intelligent partially overlapping channel assignment in sdn-iot," IEEE Communications Magazine, vol. 56, no. 9, pp. 80-86, 2018.

[10] C. Yu, J. Lan, Z. Guo, and Y. Hu, "Drom: Optimizing the routing in software-defined networks with deep reinforcement learning," IEEE Access, vol. 6, pp. 64 533-64 539, 2018.

[11] X. Huang, T. Yuan, G. Qiao, and Y. Ren, "Deep reinforcement learning for multimedia traffic control in software defined networking," IEEE Network, vol. 32, no. 6, pp. 35-41, 2018.

[12] F. Tang, Z. M. Fadlullah, B. Mao, and N. Kato, "An intelligent traffic load prediction-based adaptive channel assignment algorithm in sdn-iot: A deep learning approach," IEEE Internet of Things Journal, vol. 5, no. 6 , pp. 5141-5154, 2018.

[13] B. Mao, F. Tang, Z. M. Fadlullah, and N. Kato, "An intelligent route computation approach based on real-time deep learning strategy for software defined communication systems," IEEE Transactions on Emerging Topics in Computing, 2019.

[14] B. Mao, F. Tang, Z. M. Fadlullah, N. Kato, O. Akashi, T. Inoue, and K. Mizutani, "A novel non-supervised deep-learning-based network traffic control method for software defined wireless networks," IEEE Wireless Communications, vol. 25, no. 4, pp. 74-81, 2018.

[15] G. Li, X. Wang, and Z. Zhang, "Sdn-based load balancing scheme for multi-controller deployment," IEEE Access, vol. 7, pp. 39612-39622, 2019.

[16] A. Tavanaei, M. Ghodrati, S. R. Kheradpisheh, T. Masquelier, and A. Maida, "Deep learning in spiking neural networks," Neural Networks, 2018.
[17] H. Mostafa, "Supervised learning based on temporal coding in spiking neural networks," IEEE transactions on neural networks and learning systems, vol. 29, no. 7, pp. 3227-3235, 2017.

[18] F. P.-C. Lin and Z. Tsai, "Hierarchical edge-cloud sdn controller system with optimal adaptive resource allocation for load-balancing," IEEE Systems Journal, 2019.

[19] Y. Xu, M. Cello, I.-C. Wang, A. Walid, G. Wilfong, C. H.-P. Wen, M. Marchese, and H. J. Chao, "Dynamic switch migration in distributed software-defined networks to achieve controller load balance," IEEE Journal on Selected Areas in Communications, vol. 37, no. 3, pp. 515$529,2019$.

[20] I. Haque, M. Nurujjaman, J. Harms, and N. Abu-Ghazaleh, "Sdsense: An agile and flexible sdn-based framework for wireless sensor networks," IEEE Transactions on Vehicular Technology, vol. 68, no. 2, pp. 18661876, 2018.

[21] S. Misra, S. Bera, M. Achuthananda, S. K. Pal, and M. S. Obaidat, "Situation-aware protocol switching in software-defined wireless sensor network systems," IEEE Systems Journal, vol. 12, no. 3, pp. 2353-2360, 2017.

[22] G. M. Dias, C. B. Margi, F. C. de Oliveira, and B. Bellalta, "Cloudempowered, self-managing wireless sensor networks: Interconnecting management operations at the application layer," IEEE Consumer Electronics Magazine, vol. 8, no. 1, pp. 55-60, 2018.

[23] J. Son and R. Buyya, "Priority-aware vm allocation and network bandwidth provisioning in software-defined networking (sdn)-enabled clouds," IEEE Transactions on Sustainable Computing, 2018.

[24] B. K. Al-Shammari, N. Al-Aboody, and H. S. Al-Raweshidy, "Iot traffic management and integration in the qos supported network," IEEE Internet of Things Journal, vol. 5, no. 1, pp. 352-370, 2017.

[25] A. Taherkhani, A. Belatreche, Y. Li, and L. P. Maguire, "A supervised learning algorithm for learning precise timing of multiple spikes in multilayer spiking neural networks," IEEE transactions on neural networks and learning systems, no. 99, pp. 1-14, 2018.

[26] Y. Miao, H. Tang, and G. Pan, "A supervised multi-spike learning algorithm for spiking neural networks," in 2018 International Joint Conference on Neural Networks (IJCNN). IEEE, 2018, pp. 1-7.

[27] F. Han, R. Li, and D. Qian, "Short-term wind speed forecasting mode based on spiking neural network," in 2018 International Conference on Advanced Mechatronic Systems (ICAMechS). IEEE, 2018, pp. 359-363.

[28] J. H. Lee, T. Delbruck, and M. Pfeiffer, "Training deep spiking neural networks using backpropagation," Frontiers in neuroscience, vol. 10, p. 508, 2016.

[29] S. Matsuda, "Bpspike: a backpropagation learning for all parameters in spiking neural networks with multiple layers and multiple spikes,' in 2016 International Joint Conference on Neural Networks (IJCNN). IEEE, 2016, pp. 293-298.

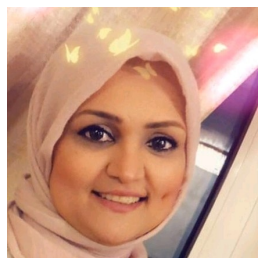

$\mathbf{N}$ adia A. SH. AL-Jamali (M'10) received a B.Sc. degree in control and systems engineering, M.Sc. degree in control engineering, and Ph.D. degree in computer engineering from the University of Technology, Baghdad, Iraq. She is currently working at Brunel University London, London, U.K. Her fields of interest are computer control, wireless sensor networks, intelligent systems, neural networks and robotics. 


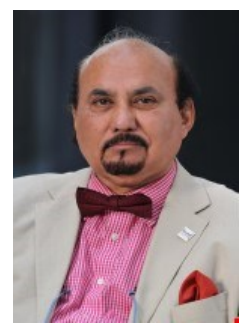

H amed S. Al-Raweshidy(SM'03) received B.Eng. and M.Sc. degrees from the University of Technology, Baghdad, Iraq, in 1977 and 1980, respectively, a Postgraduate Diploma from Glasgow University, Glasgow, U.K., in 1987, and a Ph.D. degree from the University of Strathclyde, Glasgow, in 1991, all in electronic engineering. He has worked with the Space and Astronomy Research Center, Baghdad, Perkin Elmer, Waltham, British Telecom, Oxford University, Manchester Metropolitan University, and Kent University, Canterbury, U.K. He is currently the Director of the Wireless Network and Communications Centre, Brunel University London, London, U.K. 